P. Colwell

Nagoya Math. J.

Vol. 50 (1973), 1-6

\title{
MEROMORPHIC FUNCTIONS WITH LARGE SETS OF JULIA POINTS
}

\author{
PETER COLWELL
}

1. Introduction. Let $D=\{z:|z|<1\}$ and $C=\{z:|z|=1\}$. If $W$ denotes the Riemann sphere equipped the chordal metric $X$, let $f: D \rightarrow$ $W$ be meromorphic. A chord $T$ lying in $D$ except for an endpoint $\gamma \in C$ is called a Julia segment for $f$ if for each Stolz angle $\Delta$ in $D$ at $\gamma$ which contains $T, f$ assumes infinitely often in $\Delta$ all values of $W$ with at most two exceptions. We call $\gamma \in C$ a Julia point for $f$ if every chord in $D$ ending at $\gamma$ is a Julia segment for $f$, and we denote by $J(f)$ the set of Julia points of $f$.

In this paper we show that for a certain class of functions meromorphic in $D$ the sets of Julia points are residual in $C$. This class of functions lies in the intersection of two previously-studied classes of functions. In [1] K. Barth defined the class $A_{m}: f \in A_{m}$ if $f$ is meromorphic in $D$, and if for each point $\gamma$ of a set dense in $C$ there exists a curve $K$ in $D$ ending at $\gamma$ such that $\lim _{z \rightarrow r(z \in K)} f(z)$ exists. More recently, in [7] K-F. Tse divided all functions meromorphic in $D$ into two classes in the following way. For each pair of points $z, w \in D$, the hyperbolic distance between $z$ and $w$ is defined by

$$
\rho(z, w)=(1 / 2) \log \{[1+\sigma(z, w)] /[1-\sigma(z, w)]\},
$$

where $\sigma(z, w)=|z-w| /|1-\bar{w} z|$. A meromorphic function $f$ is of the second kind if there exist a sequence $\left\{z_{n}\right\}_{n=1}^{\infty} \subset D,\left|z_{n}\right| \rightarrow 1$, a constant $r>0$, and a point $\alpha \in W$ such that for $\mathscr{D}(r)=\bigcup_{n=1}^{\infty}\left\{z: \rho\left(z, z_{n}\right)<r\right\}, f$ tends uniformly to $\alpha$ as $|z| \rightarrow 1$ in $\mathscr{D}(r)$. And $f$ is of the first kind if it is not of the second kind. Tse's results in [7] characterize the functions of the first kind and show how wild their boundary behavior must be.

THEOREM 1. If $f \in A_{m}$ is of the first kind, then $J(f)$ is residual in $C$.

Received April 11, 1972. 
After proving Theorem 1 in $\S 2$, we show that if in addition the set of points on $C$ at which $f$ has an asymptotic value is of measure $2 \pi$ on $C$, then $J(f)$ has measure $2 \pi$ on $C$ also. It is important to note that these results apply to the Tsuji functions, a well-studied class of meromorphic functions. Following the notation in [5], let $f^{*}(z)=$ $\left|f^{\prime}(z)\right| /\left(1+|f(z)|^{2}\right)$ be the spherical derivative of $f$ in $D ; f$ is a Tsuji function if for some finite constant $l>0, \sup _{r<1}\left[\int_{0}^{2 \pi} f^{*}\left(r e^{i \theta}\right) r d \theta\right]<l$. It is a consequence of [5, Theorem 6] that each Tsuji function is in class $A_{m}$.

2. Proof of Theorem 1. We begin with a few lemmas. For any $\gamma \in C$ and any $\beta \in(-\pi / 2, \pi / 2)$, let $T(\gamma, \beta)$ denote the chord in $D$ ending at $\gamma$ and making angle $\beta$ with the radius to $\gamma$.

LEMMA 1. Let $f$ be meromorphic in $D$ and suppose for some $\gamma \in C$, $\beta \in(-\pi / 2, \pi / 2)$, that $T(\gamma, \beta)$ is not a Julia segment for $f$. Then there exists $\varepsilon>0$ such that $T(\gamma, \alpha)$ is not a Julia segment for $f$ if $\alpha \in(-\pi / 2, \pi / 2)$ and $|\alpha-\beta|<\varepsilon$.

Proof. Obvious.

For each $\beta \in(-\pi / 2, \pi / 2)$, let $E(\beta)=\{\gamma \in C: T(\gamma, \beta)$ is not a Julia segment for $f$ \}.

LEMMa 2. Let $f$ be meromorphic in $D$ and $E$ be the set of points on $C$ which are not Julia points for $f$. Then $E=\cup_{\beta} E(\beta)$, where $\beta$ is rational and $\beta \in(-\pi / 2, \pi / 2)$.

Proof. If $\gamma \in E$, from Lemma 1 it follows that $\gamma \in E(\beta)$ for some rational $\beta \in(-\pi / 2, \pi / 2)$. And it is obvious that for each rational $\beta \in(-\pi / 2, \pi / 2), E(\beta) \subset E$.

For each chord $T(\gamma, \beta)$ at $\gamma \in C$, we let $\Delta(\gamma, \beta, \alpha)$ denote the Stolz angle in $D$ at $\gamma$ which is symmetric about $T(\gamma, \beta)$ and has vertex angle $\alpha$. (We presume here that $0<\alpha<\pi / 2-|\beta|$.)

Lemma 3. Let $\beta, \alpha$ be fixed, where $\beta \in(-\pi / 2, \pi / 2)$ and $0<\alpha<\pi / 2$ $-|\beta|$. If $M=\tanh ^{-1}\{\sin (\alpha / 2) /[4+\sin (\alpha / 2)]\} \quad$ and $z \in T(1, \beta)$, then $\{w \in D: \rho(w, z)<M\} \subset \Delta(1, \beta, \alpha)$.

Proof. From a lemma of P. Lappan [7, Lemma 2], if $\rho(z, w)<M$, then $|w-z| /(1-|z|) \leq 2 \tanh M /(1-\tanh M)=(1 / 2) \sin (\alpha / 2)<\sin (\alpha / 2)$. Thus $|w-z|<(1-|z|) \sin (\alpha / 2) \leq|1-z| \sin (\alpha / 2)$, and $w \in \Delta(1, \beta, \alpha)$. 
Now suppose $f \in A_{m}$ is of the first kind but $J(f)$ is not residual. Then $C-J(f)$ is of second category on $C$, and Lemma 2 implies that for some rational $\beta \in(-\pi / 2, \pi / 2), E(\beta)$ is of second category on $C$.

For each positive integer $n$, let

$$
\begin{gathered}
E_{n}(\beta)=\{\gamma \in E(\beta): \\
\exists \alpha \geq 1 / n \ni f \text { omits at least three values }\} . \\
\text { in } \Delta(\gamma, \beta, \alpha)
\end{gathered}
$$

We see that $E_{n+1}(\beta) \supset E_{n}(\beta)$, and $E(\beta)=\bigcup_{n} E_{n}(\beta)$. Thus for some integer $N, E_{N}(\beta)$ is of second category on $C$.

Since, for each $\gamma \in E_{N}(\beta), f$ omits at least three values in $\Delta(\gamma, \beta, 1 / N)$, there exists $d(\gamma)>0$ with this property: for any two sets $A, B$ on $W$ whose union contains the values omitted by $f$ in $\Delta(\gamma, \beta, 1 / N)$, either $\operatorname{diam} A \geq d(\gamma)$, or $\operatorname{diam} B \geq d(\gamma)$. For each positive integer $j$, let $E_{N, j}(\beta)=$ $\left\{\gamma \in E_{N}(\beta): d(\gamma) \geq 1 / j\right\}$. Clearly $E_{N, j+1}(\beta) \supset E_{N, j}(\beta)$, and $E_{N}(\beta)=\bigcup_{j} E_{N, j}(\beta)$. Hence, for some integer $J>0, E_{N J}(\beta)$ is of second category on $C$.

If $\gamma \in E_{N J}(\beta)$, there exists $\mu>0$ such that $f$ never assumes some three distinct values in the region $\Delta(\gamma, \beta, 1 / N) \cap\{|z-\gamma|<\mu\}$. For each positive integer $k$, let $E_{N J, k}(\beta)=\left\{\gamma \in E_{N J}(\beta): \mu \geq 1 / k\right\}$. Since $E_{N J, k+1}(\beta)$ $\supset E_{N J, k}(\beta)$ and $E_{N J}(\beta)=\bigcup_{k} E_{N J, k}(\beta)$, there exists integer $K>0$ such that $E_{N J K}(\beta)$ is of second category on $C$. For brevity let us denote $E_{N J K}(\beta)$ by $E^{*}$.

There exists an arc $A$ on $C$ such that $E^{*}$ is dense in $A$. Since $f \in A_{m}$, we can choose an interior point $\lambda \in A$ at which there ends a curve $\Gamma$ in $D$ along which $f$ has a limit. Let $\Gamma^{\prime}$ be the "last part" of $\Gamma$ in $\{|z-\lambda|<1 / K\}$ and $\left\{w_{m}\right\}$ be any sequence on $\Gamma^{\prime}$ converging to $\lambda$. For each $m$, let $\varepsilon(m)=2^{-m}$, and let $\delta(m)>0$ be chosen so that $X\left[f(w), f\left(w_{m}\right)\right]$ $<\varepsilon(m)$ whenever $\rho\left(w, w_{m}\right)<\delta(m)$. For $m$ sufficiently large there exists $\gamma_{m} \in E^{*}$ for which the chord $T\left(\gamma_{m}, \beta\right)$ intersects the neighborhood $\left\{w \in D: \rho\left(w, w_{m}\right)<\delta(m)\right\}$. We select a point $v_{m} \in T\left(\gamma_{m}, \beta\right)$ (which may be $w_{m}$ itself) such that $\left|v_{m}-\gamma_{m}\right|<1 / K$ and $\rho\left(v_{m}, w_{m}\right)<\delta(m)$. Within each such neighborhood $\left\{w \in D: \rho\left(w, w_{m}\right)<\delta(m)\right\}$ we deform $\Gamma^{\prime}$ to make it pass through $v_{m}$. The resulting curve we call $\Gamma^{*}$, and we see that $f$ has a limit as $z \rightarrow \lambda$ along $\Gamma^{*}$.

Since $f$ is of the first kind, $\left\{v_{m}\right\}$ is a $\rho$-sequence [7, Corollary 3.1]. Thus for each $r>0$, [4, Theorem 2] implies that there exist sets $G(m, r)$, $H(m, r)$ on $W$ with chordal diameter less than or equal to $r$, and integer $M(r)>0$ such that for $m>M(r)$ 


$$
W-[G(m, r) \cup H(m, r)] \subset f\left[\left\{w \in D: \rho\left(w, v_{m}\right)<r\right\}\right] .
$$

We choose $r$ to be less than the smaller of $1 / J$ and $\tanh ^{-1}\{\sin (1 / 2 N) /$ $[4+\sin (1 / 2 N)]\}$. For this choice of $r$ and every $m>M(r)$ we have:

(i) $\left\{w \in D: \rho\left(w, v_{m}\right)<r\right\} \subset \Delta\left(\gamma_{m}, \beta, 1 / N\right)$ by Lemma 3;

(ii) $f\left[\left\{w \in D: \rho\left(w, v_{m}\right)<r\right\}\right]$ omits at least three values, all of which lie in $G(m, r) \cup H(m, r)$.

But since $\gamma_{m} \in E^{*} \subset E_{N J}(\beta)$, either $\operatorname{diam} G(m, r) \geq 1 / J>r$, or $\operatorname{diam} H(m, r)$ $\geq 1 / J>r$. This is a contradiction. Hence $J(f)$ is residual on $C$.

3. Further results. Now we consider functions meromorphic in $D$ which have asymptotic values at almost every point of $C$.

THEOREM 2. Suppose $f$ is meromorphic in $D$ and has an asymptotic value at each point of a set of measure $2 \pi$ on $C$. If $f$ is of the first kind, then, $J(f)$ is residual and of measure $2 \pi$ on $C$.

Proof. That $J(f)$ is residual follows from Theorem 1. If $C-J(f)$ has positive measure on $C$, we can easily alter the selection process in the proof of Theorem 1 so that the resulting set $E^{*}$ has positive measure on $C$. And at every point of some subset of $E^{*}$ of positive measure $f$ has an asymptotic value.

Let $\lambda \in E^{*}$ be a two-sided accumulation point of $E^{*}$ at which $f$ has an asymptotic value. The remainder of the argument proceeds as in the proof of Theorem 1: we construct a curve ending at $\gamma$ along which $f$ has a limit, and which intersects a sequence of chords $\left\{T\left(\gamma_{m}, \beta\right)\right\}$, where $\gamma_{m} \in E^{*}$ and $\gamma_{m} \rightarrow \lambda$.

Since Tsuji functions of the first kind satisfy the hypotheses of both Theorems 1 and 2, we have a corollary.

COROLLARY 1. If $f$ is a Tsuji function of the first kind, $J(f)$ is residual and of measure $2 \pi$ on $C$.

For each $\alpha \in D$, let $\phi_{\alpha}(z)=(z-\alpha) /(1-\bar{\alpha} z)$. In [3] Collingwood and Piranian defined the Tsuji set of a meromorphic function $f$ to be the set of points $\alpha \in D$ such that $f \circ \phi_{\alpha}$ is a Tsuji function. The following lemma, whose proof we omit, permits a slight extension of Corollary 1.

LEMMA 4. Let $f$ be meromorphic in $D$ and $\alpha \in D$. Then $f$ has $a$ Julia point at $\gamma \in C$ if and only if $f \circ \phi_{\alpha}$ has a Julia point at $\phi_{\alpha}^{-1}(\gamma)$. 
COROLLARY 2. If $f$ is a meromorphic function of the first kind with nonempty Tsuji set, then $J(f)$ is residual and of measure $2 \pi$ on $C$.

4. Some examples. The author is grateful to Professor $K-F$. Tse for Example 1, which exhibits a function satisfying the hypothesis of the theorems and corollaries above.

EXAMPLE 1. There exists a Tsuji function of the first kind. Let $\Lambda$ be a monotone spiral in $D$ with the property that for any $\theta \in[0,2 \pi]$, if $\left\{z_{n}(\theta)\right\}_{n=1}^{\infty}=\Lambda \cap\{z \in D: \arg z=\theta\}$ then $\rho\left[z_{n}(\theta), z_{n+1}(\theta)\right] \rightarrow 0$ as $n \rightarrow \infty$. Select the monotone sequence $\left\{w_{n}\right\}_{n=0}^{\infty}$ from $\Lambda$ with $w_{0}=0$ and $\rho\left(w_{n}, w_{n+1}\right)$ $=1 / n$ for $n \geq 1$. Let $\left\{r_{n}\right\}$ be a sequence of positive numbers such that $r_{n}+r_{n+1}<\left|w_{n+1}\right|-\left|w_{n}\right|$ for each $n$, and $r_{n}=0\left(1-\left|w_{n}\right|\right)$ as $n \rightarrow \infty$.

If $\left\{a_{n}\right\}$ is a sequence of positive numbers such that $a_{n}<r_{n}^{3}$, it is shown in [3] that $f(z)=\sum_{n}\left[a_{n} /\left(z-w_{n}\right)\right]$ is a Tsuji function with these properties: (i) if $D_{n}=\left\{z:\left|z-w_{n}\right|<r_{n}\right\}$, the series for $f$ converges uniformly in the plane less $\bigcup_{n} D_{n}$; (ii) if $\left\{w_{k}\right\}$ is a subsequence of $\left\{w_{n}\right\}$ such that $w_{k} \rightarrow \gamma \in C$, for $k$ sufficiently large the values $f$ omits in $D_{k}$ lie in arbitrarily small neighborhoods of $f(\gamma)$.

Now let $\lambda \in C$, let $\left\{\xi_{n}\right\}$ be a sequence in $D$ with $\xi_{n} \rightarrow \lambda$, and let $\delta>0$ be fixed. For each integer $k$, if $z \in D_{k}, \sigma\left(z, w_{k}\right) \leq r_{k} /\left(1-\left|w_{k}\right|\right)$, so $\sigma\left(z, w_{k}\right)$ $\rightarrow 0$ and $\rho\left(z, w_{k}\right) \rightarrow 0$ as $k \rightarrow \infty$. Hence $\mathscr{D}=\bigcup_{n}\left\{z \in D: \rho\left(\xi_{n}, z\right)<\delta\right\}$ contains infinitely many disks $D_{k}$, and $f$ cannot tend to a constant limit as $|z| \rightarrow 1$ in $\mathscr{D}$. Therefore $f$ is of the first kind.

EXAMPLE 2. There exists a Tsuji function $f$ of the second kind such that $J(f)=C$. This example is due to Collingwood and Piranian [3, Theorem 1]; here we show additionally that the function is of the second kind.

Let $z_{n}=\left(1-n^{-1 / 2}\right) \exp (i \log n), n=2,3,4, \cdots$, and let $\left\{r_{n}\right\}_{n=2}^{\infty},\left\{a_{n}\right\}_{n=2}^{\infty}$ be sequences of positive numbers such that $r_{n}+r_{n+1}<\left|z_{n+1}\right|-\left|z_{n}\right|, 0<a_{n}$ $<r_{n}^{3}$. Then ([3, Theorem 1]) $f(z)=\sum_{n=2}^{\infty}\left[a_{n} /\left(z-z_{n}\right)\right]$ is a Tsuji function such that $J(f)=C$.

The sequence $\left\{z_{n}\right\}$ lies on the monotone spiral

$$
\Gamma:\left\{z(t)=\left(1-t^{-1 / 2}\right) \exp (i \log t): 1 \leq t<\infty\right\} .
$$

For any $\theta \in[0,2 \pi]$, if $\left\{w_{k}(\theta)\right\}_{k=1}^{\infty}=\Gamma \cap\{z: \arg z=\theta\}$ is arranged in order of increasing modulus, direct calculation shows that $\lim _{k \rightarrow \infty} \rho\left[w_{k}(\theta), w_{k+1}(\theta)\right]$ $=\pi / 2$. 
Choose a monotone sequence $\left\{\xi_{k}\right\}_{k=1}^{\infty}$ on the radius to $e^{i \theta}$ such that $\xi_{k}$ is midway between $w_{k}(\theta)$ and $w_{k+1}(\theta)$, so that $\xi_{k} \rightarrow e^{i \theta}$. We can choose a number $\delta, 0<\delta<\pi / 2$, and positive integer $K$ such that

$$
\mathscr{D}(\delta, K)=\bigcup_{k \geq K}\left\{z \in D: \rho\left(z, \xi_{k}\right)<\delta\right\}
$$

is disjoint from all the disks $\left\{z \in D: \rho\left(z, z_{n}\right)<r_{n}\right\}$. The details of $[3$, Theorem 1] show that $f$ converges uniformly to a constant as $|z| \rightarrow 1$ in $\mathscr{D}(\delta, K)$. Thus $f$ is of the second kind.

If $E$ is a subset of $C$ which is residual but of measure 0 on $C$, there exists a Tsuji function $g$ of bounded characteristic such that $E \subset J(g)$ [3, Theorem 3]. Thus $J(g)$ is residual and of measure 0 on $C$. And there exists a Tsuji function $h$ for which $J(h)$ is of measure $2 \pi$ but of first category on $C$ [2, pp. 199-200]. Both $g$ and $h$ are necessarily of the second kind.

\section{REFERENCES}

[1] Barth, K., Asymptotic values of meromorphic functions, Mich. Math. J., 13 (1966), 321-340.

[ 2 ] Collingwood, E. F., A boundary theorem for Tsuji functions, Nagoya Math. J., 29 (1967), 197-200.

[3] - and G. Piranian, Tsuji functions with segments of Julia, Math. Z., 84 (1964), 246-253.

[ 4 ] Gauthier, P., A criterion for normalcy, Nagoya Math. J., 32 (1968), 277-282.

[5] Hayman, W. K., The boundary behavior of Tsuji functions, Mich. Math. J., 15 (1968), 1-26.

[6] Lappan, P., Some sequential properties of normal and non-normal functions with applications to automorphic functions, Comm. Math. Univ. Sancti Pauli, 12 (1964), 41-57.

[7] Tse, K.-F., Some results on value distribution of meromorphic functions in the unit disk, Nagoya Math. J., 34 (1969), 105-119.

Department of Mathematics

Iowa State University 